Supporting information to

\title{
Theoretical Investigation of the First-Shell Mechanism of Nitrile Hydratase
}

\author{
Kathrin H. Hopmann ${ }^{\dagger}$, Jing-Dong Guo ${ }^{\#}$, Fahmi Himo ${ }^{\dagger, *}$
}


A

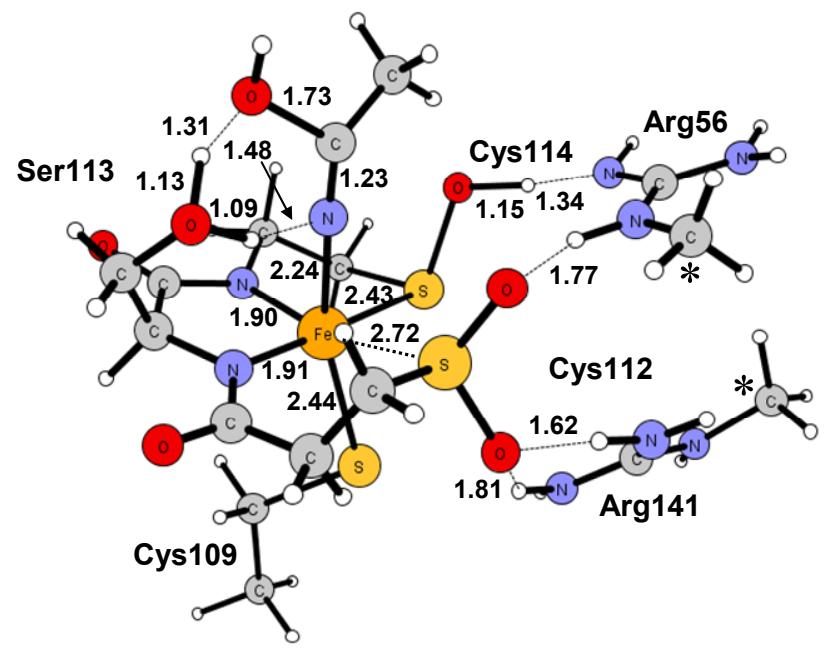

B

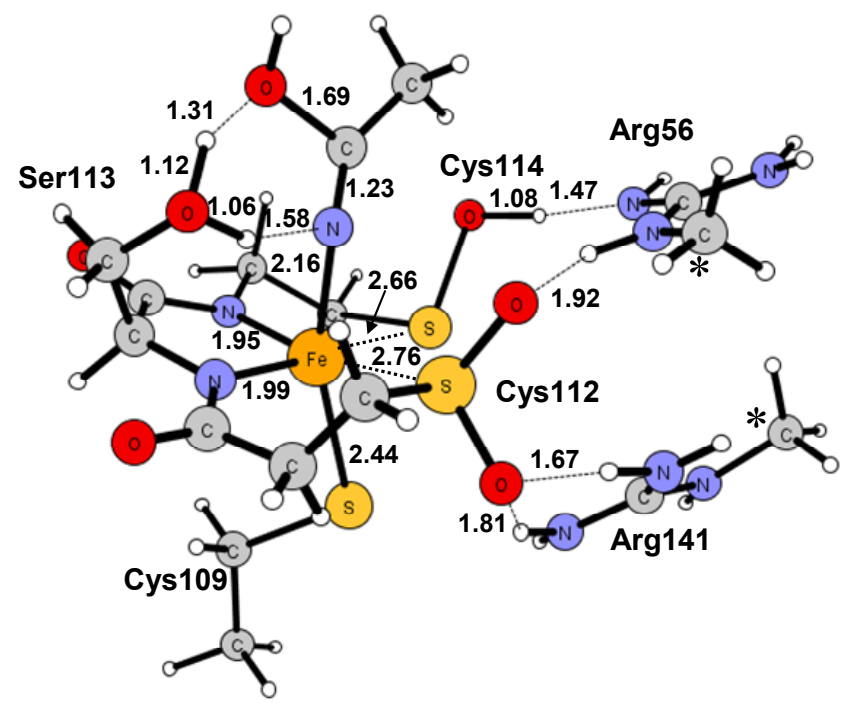

Figure S1. Transition state of Model B in A) the quartet state and B) the sextet state.

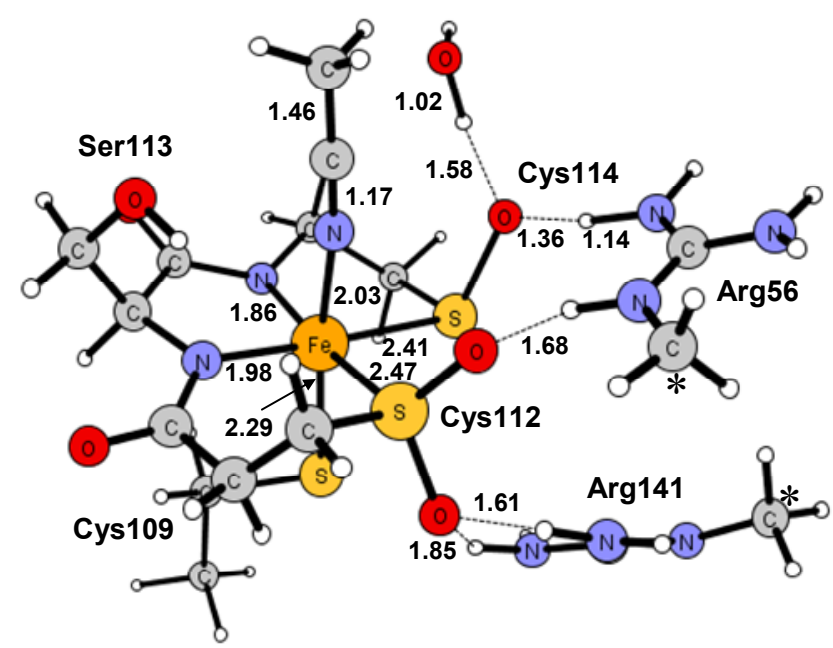

Figure S2. Reactant of Model B with water hydrogen bonded to Cys114-SO- 